\title{
Éléments sur la collection Moriceau
}

\section{Gilles Bounoure}

\section{(2) OpenEdition}

\section{Journals}

Édition électronique

URL : http://journals.openedition.org/jso/7180

DOI : $10.4000 /$ jso. 7180

ISSN : $1760-7256$

Éditeur

Société des océanistes

Édition imprimée

Date de publication : 15 décembre 2014

Pagination : 225-232

ISBN : 978-2-85430-118-2

ISSN : 0300-953x

\section{Référence électronique}

Gilles Bounoure, «Éléments sur la collection Moriceau », Journal de la Société des Océanistes [En ligne], 138-139 | 2014, mis en ligne le 15 décembre 2014, consulté le 02 mai 2019. URL : http:// journals.openedition.org/jso/7180; DOI : 10.4000/jso.7180 


\section{MISCELLANÉES}

\section{Éléments sur la collection Moriceau}

par

\section{Gilles BOUNOURE*}

Le riche et beau catalogue conçu par Roger Boulay et Emmanuel Kasarhérou pour accompagner la récente exposition Kanak. L'art est une parole qu'ils ont présentée au musée du quai Branly sur les arts de Nouvelle-Calédonie mentionne à quatre reprises :

"[des] objets de la collection de Jules-Édouard Moriceau (1846-après 1902) [...] administrateur colonial et chef des Affaires indigènes en NouvelleCalédonie de 1875 à 1897. Ancienne collection du musée Umlauff à Hambourg, Staatliche Museen zu Berlin, Ethnologisches Museum, Berlin. " (Kasarhérou, in Boulay et Kasarhérou, $2013: 205$; voir aussi $262,263,320)$

Dans le catalogue de la dernière grande exposition d'art kanak organisée en France, De Jade et de nacre, Boulay livrait déjà ces éléments :

"Moriceau Édouard: ancien officier de Marine, il est nommé chef du $4^{\mathrm{e}}$ arrondissement en 1896 (Hienghène-Touho-Koné-Témala). Acteur de la politique de cantonnement, il devient chef du service des Affaires indigènes. Il fait don de cent cinquante et un objets au musée de Berlin (1895).» (1990 : 227)

C'est à tenter de préciser la personnalité de ce collecteur et le parcours européen des objets qu'il a recueillis - et non pas "donnés " par la suite, comme estimé en 1990, mais bel et bien vendus comme le suggère le catalogue de 2013 - que sont employés les développements qui suivent.

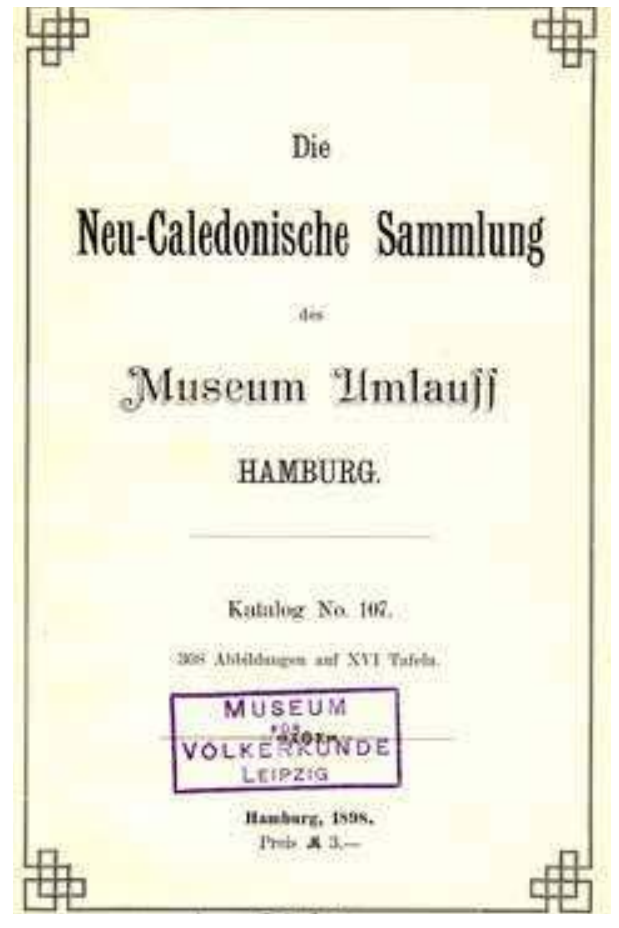

Document 1. - Catalogue Umlauff, page de titre (Courtesy MfV Leipzig)

La note précitée d'E. Kasarhérou complète l'exposé qu'il consacre aux "Kanak à l'Exposition universelle de 1889 à Paris " (pp. 203205 ) indiquant que "de nombreux objets kanak» réunis pour cette manifestation "ali-

* gillesbounoure@gmail.com 
mentèrent les musées européens, comme les musées de Berlin ou de Stuttgart ", tandis que sa note suivante se réfère à " deux pièces [...] aujourd'hui au Linden Museum de Stuttgart (inv. 67845 et 67846, coll. du Dr H. Meyer ", repérables sur certains des dessins de presse publiés à l'occasion de cette exposition. Or divers écrits et documents visuels laissent penser avec suffisamment de vraisemblance que la plupart de ces objets, ainsi que d'autres non mentionnés dans le catalogue de R. Boulay et E. Kasarhérou, proviennent de la " collection Moriceau » et ont été acquis en Europe à l'occasion de cette même Exposition - celle-là même qui décida Festetics de Tolna à aller à son tour collecter des objets dans le Pacifique, pour mentionner une autre mémorable et méritoire enquête-exposition de R. Boulay.

À l'occasion d'échanges sur des objets anciennement au musée de Leipzig circulant sur le marché de l'art, Marion Melk-Koch, conservatrice des collections océaniennes et australiennes du Museum für Völkerkunde de Leipzig - sans qui cet article n'aurait pas vu le jour - m'a fait connaître un précieux document publié à petit nombre, Die Neu-Caledonisch Sammlung des Museum Umlauff Hamburg, sept pages d'introduction et cinquante pages de notices suivies de seize planches réunissant 308 reproductions, édité en 1898 , et conservé dans la bibliothèque de ce musée saxon parce que c'est là qu'aboutirent la plupart de ces objets $(581 \mathrm{au}$ total, nombre très supérieur aux acquisitions du musée de Berlin). Le jeune Heinrich Umlauff (1869-1925) indiquait dans son avertissement, signé du nom de la firme :

" La collection elle-même ne sera vendue que d'un seul tenant, ou à la rigueur en deux lots, si les acquéreurs en sont d'accord "

se référant à ce qui était advenu à sa première collection néo-calédonienne, dont une partie avait rejoint le musée d'ethnographie de Berlin, et le reste le Field Museum de Chicago ; ainsi s'explique que seule une poignée de musées dispose aujourd'hui de ce catalogue. Umlauff précisait aussi qu'à quelques pièces près l'ensemble venait d'un "administrateur français de haut rang » et que " pour la plupart des notices traitant successivement des objets, [c'était] Madame Moriceau [qui avait eu] la bonté de les fournir, ainsi que les matériaux documentaires mis en œuvre dans l'introduction " du catalogue, avant de rendre hommage à sa "Liebenswürdigkeit". $\mathrm{Au}$ chapitre des remerciements, s'il y a peut-être lieu d'adjoindre Umlauff pour avoir reproduit, même à titre d'argument de vente, les notices de "Frau Moriceau ", il faut en tout cas rendre grâces à M. Melk-Koch d'avoir exhumé ce catalogue dont l'intérêt ethnographique est considérable, avec des observations parfois de première main, comme il sera suggéré en conclusion.

Que peut-on savoir aujourd'hui de cet administrateur Moriceau? À défaut d'archives privées jusqu'à présent introuvables - le patronyme est commun à beaucoup de familles de la "métropole" et de la Grande Terre, ce fut également celui de condamnés au bagne et de Communards relégués avec lesquels l'administrateur n'eût pas apprécié d'être confondu -, les données publiques sont suffisantes pour reconstituer son parcours. Des documents récapitulatifs de la Légion d'Honneur (LH/1937/29) et de l'École navale, où il entra en octobre 1862, le font naître le 22 janvier 1846 à Strasbourg, de parents commerçants (s'il était germanophone, ses transactions avec Umlauff en furent certainement facilitées). Aspirant en octobre 1865, il passait enseigne de vaisseau deux ans plus tard, naviguait successivement sur le Jean Bart (vaisseau-école), l'Isis (frégate à voiles de 42 canons, chargée de convoyer des bagnards en Nouvelle-Calédonie), l'Iphigénie (frégate à voiles de 50 canons chargée du même service), le Chevert (brick affecté à la station du Pacifique), l'Alceste (frégate à voiles de 52 canons convoyant aussi des bagnards), prenait part aux combats $\mathrm{du}$ siège de Paris depuis le fort d'Ivry, et rembarquait à bord du transport Finistère (transport-écurie à propulsion mixte affecté à la déportation des anciens Communards) avant d'obtenir un congé de trois ans sans solde en novembre 1872 et de démissionner de la Marine en février 1874.

Écrit et daté du 5 novembre 1902 de la main même de l'intéressé, le "résumé des services de M. Moriceau René Jules Édouard, administrateur en chef de $2^{\mathrm{e}}$ classe des Colonies, chevalier de la Légion d'Honneur" ne fait part de son entrée dans l'administration coloniale qu'en janvier 1881, en qualité d' «adjoint d'arrondissement en Nouvelle-Calédonie ", devenant en 1884 "chef d'arrondissement", en 1887 "administrateur des Colonies" de $2^{\mathrm{e}}$ classe, puis de $1^{\text {ère }}$ classe en 1889 (on verra plus loin les raisons possibles de cette promotion rapide), "administrateur principal " en 1894, " administrateur de $2^{\mathrm{e}}$ classe nouvelle formation en Nouvelle-Calédonie » en 1896, et finalement, à Madagascar, " administrateur de 1 ère classe » en 1899 et "administrateur en chef de $2^{\text {e }}$ classe " en 1902. D'autres documents, sur lesquels on ne s'étendra pas puisqu'ils n'intéressent aucunement la Nouvelle-Calédonie, permettent de suivre sa promotion à la première classe en 1904, après avoir reçu les éloges de Lyautey pour son administration à Madagascar, et ses activités au moins jusqu'en 1919, où il rédigeait un "Projet de mission en Alsace-Lorraine " pour faire "des conférences économiques sur les colonies et préconiser l'établissement de relations directes » entre les colonies et l'Alsace- 


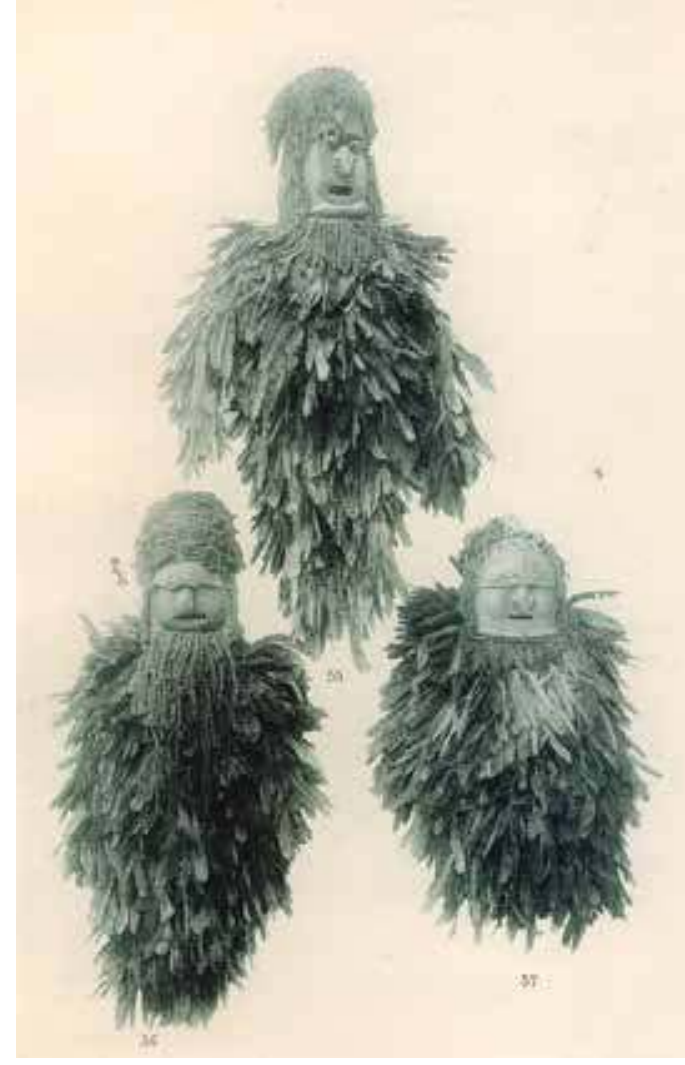

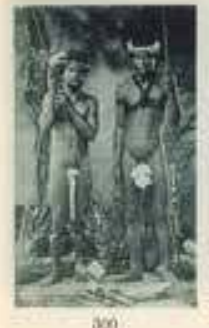
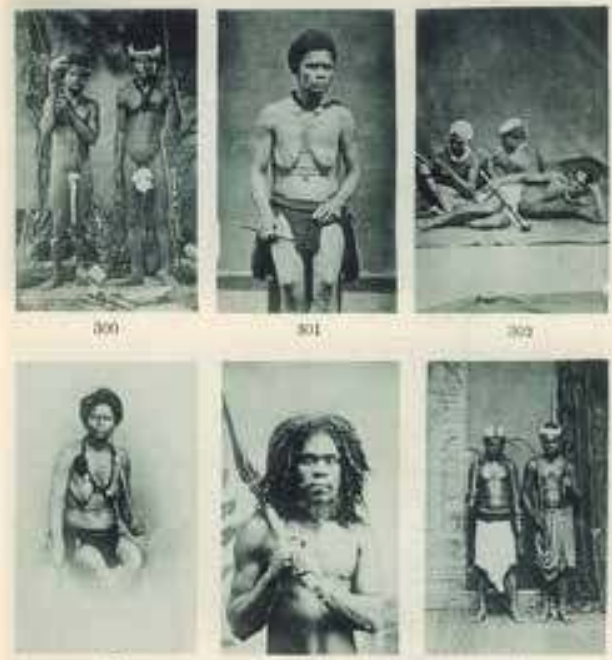

3031

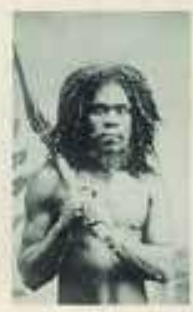

206

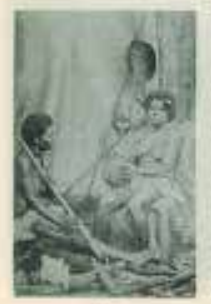

306

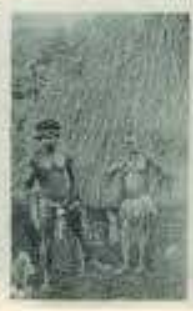

$5 y$

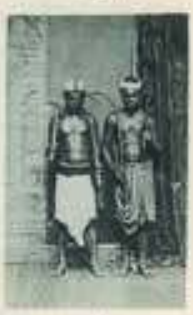

ous

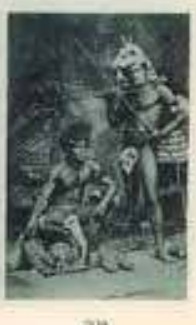

Documents 2-3. - Catalogue Umlauff, planches III et XVI (Courtesy MfV Leipzig)

Lorraine (Archives nationales d'outre-mer, ANOM 50COL74bis). Néanmoins, qu'on ait chargé un " administrateur des Colonies " d'aller porter la bonne parole de la France en Alsace-Lorraine en dit long sur les conceptions de l'époque. Le document ne dit rien de la "langue de contact ", français, allemand, elsässisch...

Entre 1872 ou 1874 et son admission en 1881 dans l'administration coloniale, il y a de fortes raisons de supposer que Moriceau s'était établi en Nouvelle-Calédonie, où l'avaient conduit à plusieurs reprises les vaisseaux de l'État. Qui d'autre pouvait-ce être que ce «M. Moriceau, ancien officier de Marine ", qui, en juillet 1878, "avait amené [...] à Bouloupari, comme Boutan à Uaraï, un certain nombre de volontaires à cheval qui l'avaient pris pour chef ", selon ce qu'en raconte le commandant Rivière - marin lui-même - qui évoque ensuite les "cavaliers Moriceau» occupant avec la troupe le pénitencier de Nouméa et à nouveau les " cavaliers Moriceau » accompagnant Servan et ses soldats pour brûler des villages kanak (Rivière, 1881 : 170, 172, 176) ? Comme l'avait relaté plus tôt La Revue des Deux Mondes (1878 : 681) :

"quarante citadins et squatters, habiles cavaliers, pourvus d'excellents chevaux, s'offrirent pour établir un service d'éclaireurs ou d'estafettes en cas de rupture des fils électriques. Leurs services furent accep- tés avec un vif empressement. Leurs patrouilles, leur apparition soudaine au milieu des Canaques révoltés, ont fait merveille. MM. Boutan, Moriceau et de Sonneville commandent ces hardis volontaires. »

On se demande forcément par quels moyens, doux ou rudes, procédant de la transaction ou relevant de la rapine et de l'abus d'autorité, le couple Moriceau en vint à constituer, avant ou après la répression de la grande insurrection de 1878, une collection forte de plusieurs centaines d'objets kanak dont certains de taille impressionnante (la flèche faîtière qu'ils recueillirent à Canala et qui entra en 1895 dans les collections berlinoises, reproduite p. 205 de Kanak, l'art est une parole, est haute de $244 \mathrm{~cm}$ ). Selon les extraits qu'en ont publiés d'éminents historiens de la colonisation comme Alain Saussol (1979: 483 pour les références de ces rapports), Joël Dauphiné (1989: 190 sq.) et, plus récemment, Luc Legeard (2008), les rapports administratifs de la main de Moriceau ou le concernant révèlent une personnalité capable d'utiliser tous les moyens successivement ou concurremment, intraitable d'un côté, compatissant de l'autre, signalant en 1886 les ravages "navrants " des troupeaux des colons et le "découragement trop justifié " des horticulteurs kanak. Des chercheurs spécialisés sauront sûrement reconstituer le détail de ses faits 
et gestes en Nouvelle-Calédonie (spécialement comme " chef du bureau des Affaires indigènes ", un sujet en soi) et il convient de revenir à l'arrivée et au sort de ces objets en Europe.

On est loin de tout savoir sur la préparation de "l'exposition coloniale " associée à l'Exposition universelle de mai-octobre 1889 (et trop souvent confondue avec elle, organisations distinctes à étudier comme telles). Le couple Moriceau s'employa activement à la première. L'American Museum of Natural History conserve un manuscrit de la main de Moriceau, Dictionnaire abrégé des dialectes calédoniens : langue de Wagap ou Poua-tié, langue de Bondi ou Youanga, langue de Ponébo ou Tchiaouatch, langue de Balade ou Nialadiou, "par Éd. Moriceau, administrateur colonial de $2^{\mathrm{e}}$ classe, chef d'arrondissement en Nouvelle Calédonie ", comptant 191 feuillets (les 30 derniers restés vierges), et portant cette dédicace :

"À Monsieur Étienne, député, sous-secrétaire d'État aux colonies, Paris, 16 avril 1889.»

Ainsi les Moriceau avaient-ils fait le voyage de la "métropole", ainsi étaient-ils venus aussi avec bagages à double ou triple fond (selon la présente enquête, à compléter par qui pourra bien sûr). Eugène Étienne (1844-1921), député puis sénateur du département d'Oran, fut l'un des principaux représentants à la Chambre des députés de ce qu'on nomma «le parti colonial». Il n'est pas exclu que le ministre, d'origine modeste, d'abord employé des Messageries maritimes, ait voulu récompenser le zèle de Moriceau en accélérant sa carrière, comme l'y invitait implicitement l'envoi de ce manuscrit. On ne sait s'il fut aussi destinataire de « La Calédonienne, valse pour piano " par Éd. Moriceau [Musique imprimée, 1889], partition dont la BNF conserve un exemplaire.

Le zèle des Moriceau leur fit aussi apporter et présenter pour cette même festivité parisienne non pas une, mais deux collections, dont quelques documents ont gardé trace et qui réunissaient probablement un bon millier de pièces. Ainsi la Revue de l'Exposition universelle de 1889 (p. 91), sous la plume de Théodore de Wyzewa, parle élogieusement des "armes calédoniennes de la collection de M. Moriceau ", tandis que le " guide publié par la société des études coloniales et maritimes ", intitulé Colonies françaises et pays de protectorat à l'exposition universelle de 1889 (Paris, librairie Léopold Cerf), mentionne, parmi les "objets indigènes exposés", rien de moins que "l'exposition de M. Moriceau " (pp. 407-408), avec ce commentaire :

«La collection de M. Moriceau est surtout digne d'attention par le soin et le goût qu'un long séjour dans la colonie lui a permis d'apporter à sa réunion.
Elle comprend depuis les objets les plus menus jusqu'au corps fumé et séché d'un chef indigène, permettant ainsi d'embrasser, d'un coup d'œil, les habitudes et les mœurs de ces peuplades primitives. »

La même publication indique à propos de la Nouvelle-Calédonie (p. 114) :

«Parmi les produits de la mer, l'Administration expose de belles nacres, dont quelques-unes gravées, et Mme Moriceau a réuni une riche collection de coquillages, parmi lesquels ceux qui servent de monnaie aux indigènes. »

Des caricatures et des dessins de presse contemporains le confirment, puisqu'on reconnaît par exemple dans telle planche de L'exposition pour rire tirée des dessins de Georges Coutan (reproduite dans Kanak, l'art est une parole, fig. 128 p. 206), la flèche faîtière de Berlin déjà mentionnée, un tale ou jövö finalement arrivé à Stuttgart et reproduit par Sarasin (2009 [1929]: planche 38-3), ainsi qu'une autre sculpture de grande case du musée de Berlin (Sarasin : planche 39-6).

Le "collectionnisme" du couple Moriceau a laissé d'autres traces, à l'instar de ces «trois caisses de fossiles de la province de Majunga " à Madagascar, provenant des collectes de l'administrateur colonial, parvenues en $1901 \mathrm{au}$ Muséum d'histoire naturelle de Paris (Bulletin du Muséum d'histoire naturelle, 1902 : 2). Il ne serait d'ailleurs pas hors de portée de reconstituer partiellement l'attitude de l'administrateur et de son épouse, venus à Madagascar participer à la "pacification" après l'insurrection des Melambala (1895-1898), d'après divers textes qu'ils ont publiés dans la presse coloniale, tels "Le Nord de la province de Majunga ", dans La Revue de Madagascar (1902: 521-548, signé L. Moriceau, Madame parlant au nom de Monsieur, avec photographies) ou "Quelles entreprises peut-on tenter à Madagascar ?" (1903 : 195218, signé ès-qualité par l'administrateur). Quant aux collections qu'ils expédiaient, ce serait un "anachronisme psychologique", comme disait le grand et subtil historien Salvador de Madariaga, que de se demander aux frais de qui, l'administration coloniale et ses cadres de haut rang étant également disposés à l'époque à payer l'envoi de spécimens devant alimenter la "science occidentale " et soutenir le bien-fondé de la colonisation. Il s'agissait de prestige "institutionnel », aussi bien collectif qu'individuel, professionnel que personnel, c'était alors relativement peu coûteux (sans les précautions actuelles d'emballage et de manutention), et à force d'apports de ce genre aux collections publiques, il résultait de ces collaborations d'étonnantes osmoses entre scientifiques haut placés et administrateurs de 
premier rang, avec des connivences intellectuelles et politiques dont témoignent les revues savantes de l'époque.

Devant regagner son poste en NouvelleCalédonie après ce congé à Paris le temps de l'exposition, Moriceau ne pouvait guère envisager de " rapatrier" (comme on dirait aujourd'hui) ses collections. Il n'est pas exclu qu'il les ait proposées à Ernest-Théodore Hamy, mais on sait que faute de moyens, la salle océanienne du musée du Trocadéro resta fermée de 1889 (précisément) à 1910. C'est alors, dans des circonstances encore à préciser, qu'intervinrent les représentants de la firme hambourgeoise Umlauff, créée en 1868, et pour laquelle 1889 fut une année cruciale : le fondateur, Johann Friedrich Gustav (né en 1833, d'abord charpentier de marine) mourait alors que décision avait été prise d'ouvrir un musée privé à son nom. Son épouse, née Catherine Karoline Hagenbeck, prit alors la direction de la société, secondée par ses fils Heinrich (1869-1925) et Johannes (1874-1951), le premier se spécialisant dans le commerce des objets ethnographiques, le second, taxidermiste de formation, dans celui des spécimens d'histoire naturelle. Prénommé comme son grand-père, le fils aîné (1893-1974) d'Heinrich prit sa succession et continua de vendre des objets jusque dans les années 1950 . Mais Heinrich fut aussi le collaborateur de son oncle Carl Hagenbeck, marchand d'animaux exotiques, exploitant de zoos et entrepreneur de spectacles itinérants, à qui il fournissait décors et accessoires. Son nom apparaît aussi comme décorateur, mais également comme acteur et réalisateur, dans l'histoire des débuts du cinéma allemand.

Il est revenu à Hilke Thode-Arora (1989, 1992, 1997, voir aussi 2001: 21 et Glenn Penny 2002: 104 et notes) d'éclairer la part prise par la famille Umlauff et ses alliés Hagenbeck dans ce qu'on a pu dénommer plus récemment "les zoos humains". Selon l'enquête de Philippe David sur les « exhibitions zoo-ethnologiques du Jardin d'Acclimatation" du Bois de Boulogne, proposée en ligne par cette institution même, les Hagenbeck ont livré :

" onze des dix-huit premières troupes présentées par le Jardin au cours de ses quatorze premières années, de 1877 à 1891 (y compris des faux "Dahoméens"). "

Il ressort de ce travail que si les "Lapons " présentés en 1889 ne provenaient pas des Hagenbeck, ils furent bien ceux qui procurèrent les spectacles des deux années suivantes. Qu'ils aient ou non profité de l'exposition de 1889 pour y présenter ailleurs une des très nombreuses "exhibitions zoo-ethnologiques " qui se déployaient jusque dans les rues de la capitale, Carl Hagenbeck et son neveu Heinrich Umlauff étaient des familiers de Paris, et c'est probablement dans cette ville, pendant ou après l'exposition, qu'ils repérèrent la collection Moriceau et en devinrent acquéreurs, l'aisance linguistique des uns et des autres contribuant à faciliter les transactions, mais aussi la transmission des données ethnographiques concernant les objets qu'offre le catalogue de 1898.

Des recherches ultérieures permettront probablement de retracer les détails de la dispersion de la collection Moriceau après son entrée dans les stocks du "musée " Umlauff. On se bornera ici à esquisser quelques pistes. À côté des objets entrés en 1895 au musée de Berlin - ainsi qu'au Field Museum de Chicago -, si l'on veut bien tenir compte de l'avertissement du catalogue de 1898, malheureusement sans autre correspondance dans les publications contemporaines du musée américain (Annual Report..., 1898 : 295) qu'un objet isolé acquis auprès d'Umlauff en 1897-1898, un " calvarium " (crâne sans mandibule) kanak - et de ceux acquis plus tard par le Museum für Völkerkunde de Leipzig, plusieurs autres pièces (sans doute plus nombreuses que celles mentionnées au début de cet article) ont rejoint le Linden Museum de Stuttgart, au titre de la " collection Dr Hans Meyer ", personnalité plusieurs fois évoquée dans ces colonnes à propos des musées d'Outre-Rhin. Rentier associé à de fructueuses entreprises, il se fit explorateur et obtint une chaire universitaire de géographie. Engagé, comme beaucoup d'autres grands bourgeois de sa catégorie, dans une lutte de prestige opposant "la science " moderne à l'autorité alors très réelle de l'ancienne aristocratie prussienne (celle du "Junker " Bismarck d'abord), il alimenta longtemps en objets le musée de Leipzig, qui lui doit nombre de ses plus prestigieuses collections (bronzes du Bénin également acquis auprès d'Umlauff, par exemple, ou encore collection d'objets de Nouvelle-Irlande récemment passée du musée de Leipzig à celui de Berlin). À ce titre, il s'estimait en rivalité avec d'autres "connaisseurs", responsables ou fondateurs de musées, ce qu'il n'était pas, animosité dont ses correspondances avec Felix Von Luschan (musée de Berlin) et Karl Graf von Linden (Stuttgart) portent trace (voir notamment Penny, 2001 : 248-250; Buschmann, 2009 : 66 sq.). S'il offrit des objets kanak de l'ancienne collection Moriceau au Linden Museum, il est probable qu'il voulait ainsi y laisser sa marque ou un signe de sa compétence exceptionnelle en ces domaines.

Du côté de ce dernier musée, les numérotations modifiées d'un inventaire à l'autre, les difficultés d'accès à ses collections et à ses archives, sa politique ancienne et prolongée de vente et d'échange d'objets pourraient compliquer les recherches. Il n'en va pas de même à Leipzig et à Berlin. Pour le premier de ces musées, il fut aisé de reconstituer avec $\mathrm{M}$. Melk-Koch d'où provenait la "sculpture à planter " Me 5563 récemment proposée sur le marché, le galeriste qui la publiait se référant au catalogue 
de 1898 précité, mais probablement sans l'avoir consulté puisqu'il ne mentionne pas la collection d'origine. Comme il ne le précise pas davantage, l'objet faisait partie d'un lot exporté en 1976 par le marchand texan et new-yorkais Everett Rassiga avec l'appui des services secrets est-allemands chargés de "réaliser " en devises occidentales des collections d'art publiques ou même privées, en usant de tous les moyens. Pour celui de Berlin, deux enquêtes, celle de Markus Schindlbeck (2012, décrite dans le jso 135) et celle plus ancienne de Caroline Eliot Zelz (1995, malheureusement négligée par M. Schindlbeck comme par R. Boulay et E. Kasarhérou), livrent quelques indications sur certaines pièces de la collection Moriceau distraites des réserves de ce musée. $M$. Schindlbeck signale ainsi une "figure d'ancêtre " cédée au Folkwang Museum d'Essen (p. 240), un masque (inventorié VI 12844) et cinq massues vendues à Speyer en 1922 (p. 244), cinq armes et une hache de pierre acquises par le même l'année suivante (pp. 246 et 251). Selon C. E. Zelz, manquent aussi à ce musée deux autres masques de même provenance (inventoriés VI 12846 et 12847), non repérés par M. Schindlbeck. Elle signale d'autre part que la firme Umlauff a vendu, de cette même collection Moriceau, deux masques au musée d'ethnographie de Hambourg, en 1896 et 1903, puis deux autres à celui de Cologne, en 1907 (1995 : 58, 60-63).

À ces données publiques, ou du moins assez bien connues des spécialistes, restera à ajouter, pour les confirmer, les infirmer ou les détailler, ce que recèlent les archives privées et publiques encore à explorer. Pour l'instant, que tirer des éléments réunis ici ? Ils permettent quelquefois de discuter assez précisément d'objets importants, voire uniques dans les collections occidentales. Par exemple Sarasin (2009: 233, texte français ici à confronter avec l'allemand de l'édition de 1929, autrement plus clair) met en doute la sincérité de la "momie d'homme en position accroupie " du musée de Berlin (qu'il reproduit planche 16-4), les cordelettes et le panier la contenant ne gardant pas de traces de la putréfaction du cadavre qui aurait pu, estime-t-il, y être enfermé beaucoup plus tard. Il s'agit très certainement du même " corps fumé et séché d'un chef indigène " qui formait l'un des fleurons de "l'exposition Moriceau " de 1889 à Paris. Il a été montré par ailleurs à propos d'autres objets océaniens (Leonie Gärtner dans Schindlbeck et al., 2010, voir JSO 135) qu’à la différence des musées de cette époque, la firme Umlauff disposait d'un service de restauration avant la lettre, utilisant des matériaux et des produits aisément identifiables et datables, de sorte qu'avec les connaissances et les techniques d'aujourd'hui, il serait aisé de vérifier si les doutes de Sarasin étaient fondés.

Pour finir, à quelques conclusions que puissent aboutir de futures recherches sur l'attitude de Moriceau en Nouvelle-Calédonie en tant que

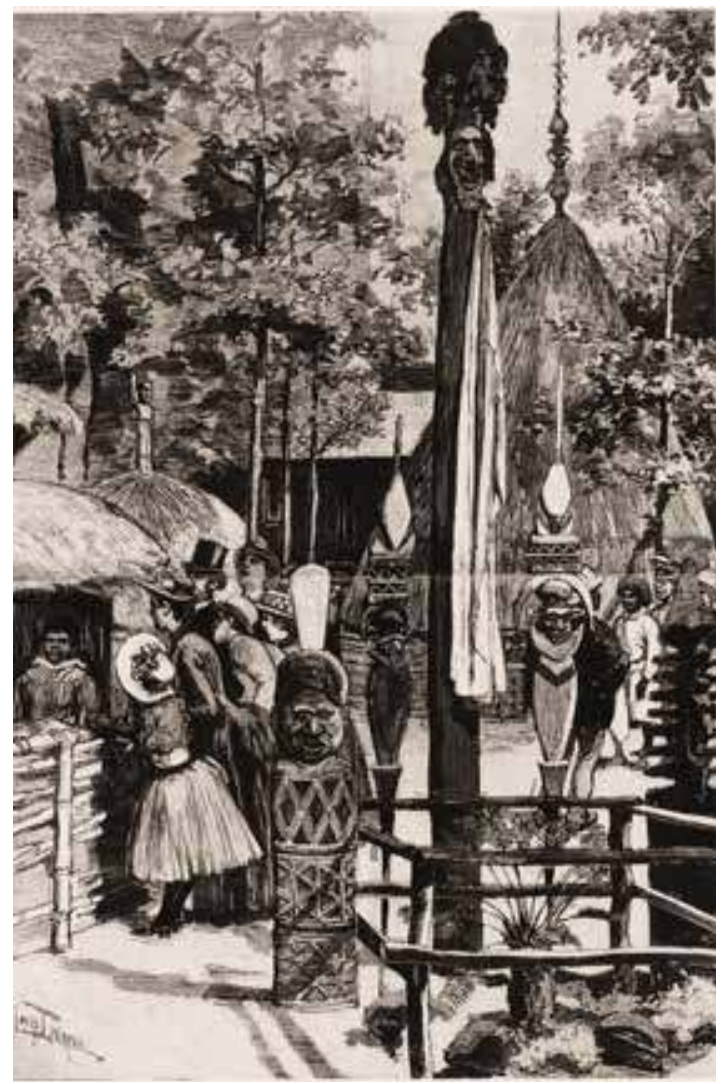

Document 4. - Le « village canaque » de l'Exposition universelle, dessin de Louis Tinayre dans Le Monde illustré du 27 juin 1889.

Au premier plan, jovo ou tale " atypique " de la collection Moriceau entré en 1895 au musée de Berlin et commenté en 1929 par Fritz Sarasin (2009: 142 , et planche 39-6).

particulier, "citadin ou squatter", puis comme fonctionnaire de l'administration coloniale et collecteur d'objets (points sur lesquels Alain Saussol a bien voulu communiquer des éléments qu'on trouvera résumés en annexe), on doit au moins lui reconnaître, ainsi qu’à son épouse, un souci, alors assez rare, de documenter les spécimens ethnographiques qu'il recueillait. Laissant à d'autres le soin de retraduire en français l'ensemble des notices du catalogue de 1898 , on se limitera à en donner trois échantillons concernant les pierres magiques (à comparer avec ce qu'en écrivirent Sarasin, 2009 : 257 sq. et Boulay $1990: 161$, et sujet abordé pp. 92, $144,221,280-282$ du catalogue de 2013). On y lit ainsi p. 3, pour le $\mathrm{n}^{\circ} 11$, reproduit pl. 2 fig. 14 :

«Pierre tabou pour un peu de pluie, galet de pierre noirâtre de moyenne grosseur. Les magiciens utilisaient ces pierres magiques pour invoquer les esprits et obtenir d'eux pluies et autres, ce pourquoi naturellement les indigènes les payaient. Antérieurement on donnait de la monnaie, aujourd'hui un litre de rhum, et si les esprits offrent une résistance particulière et se montrent têtus, on double le don. Provenance Nyacouya, une tribu de Houaïlou. » 
Page 4, pour le $\mathrm{n}^{\circ} 21$, reproduit pl. 2 fig. 24 :

«Pierre fétiche pour le tonnerre. Comme le tonnerre accompagne les orages, précède de grosses chutes de pluie et annonce la fin de la sécheresse, le magicien lui enjoint de faire pleuvoir. Pierre grisâtre, arrondie, aplatie sur une face et plutôt en forme de galet avec une fente courant tout autour. Médihouen, Houaïlou. »

Et même page, pour le n ${ }^{\circ} 25$, reproduit pl. 2 fig. 28 :

"Pierre fétiche pour le retour du soleil. Pierre en ovale allongé de couleur rougeâtre. Lorsque, durant la saison des pluies, il pleut si fort ou si continuellement que les récoltes menacent de pourrir, le magicien offre un sacrifice pour lequel il cuit des patates douces et avec ces pierres il enjoint aux esprits de laisser paraître le soleil. Médihouen. »

Sans avoir le développement des pages consacrées par Sarasin (p. 262 sq.) aux "pierres pluie ", ces exemples suffiront peut-être à convaincre que l'intérêt des collectes effectuées par les époux Moriceau excède la seule « histoire des collections " océaniennes en Occident.

\section{BIBLIOGRAPHIE SÉLECTIVE ${ }^{1}$}

ANNUAL Report of the Director to the Board of Trustees for the year 1894-95, Field Columbian Museum Publication 6, Chicago, U. S. A., October 1895, (Report $\left.n^{\circ} 1\right)$ et livraisons des quatre années suivantes.

Boulay Roger (éd.), 1990. De Jade et de nacre, Paris, Réunion des Musées nationaux.

Boulay Roger et Emmanuel Kasarhérou (éd.), 2013. Kanak. L'art est une parole, Paris-Arles, musée du quai Branly-Actes Sud.

Bulletin du Muséum national d'histoire naturelle, 1902, vol. 1.

Buschmann F. Rainer, 2009. Anthropology's Global Histories: The Ethnographic Frontier in German New Guinea, 1870-1935, Honolulu, University of Hawaii Press.

Dauphiné Joël, 1989. Les spoliations foncières en Nouvelle-Calédonie, Paris, L'Harmattan.

GärTNER Leonie, 2010. Restaurierung der Objekte der Sammlung Hans Meyer am Ethnologischen Museum, in Markus Schindlbeck (éd.), Die Hans Meyer-Sammlung Bismarck-Archipel, Berlin, Staatliche Museen zu Berlin, pp. 55-65.
Legeard Luc, 2008. L'affaire d'Ina, Tieti, Poindimié, in Sylvette Boubin-Boyer (éd.) Révoltes, conflits et guerres mondiales en Nouvelle-Calédonie et dans sa région, Paris, L'Harmattan, pp. 65-86.

Penny H. Glenn, 2001. Objects of Culture. Ethnology and Ethnographic Museums in Imperial Germany, Chapel Hill, University of North Carolina Press.

La Revue de Madagascar, 1902 (2 $2^{\mathrm{e}}$ semestre), 1903 ( $1^{\text {er }}$ semestre).

Rivière Henri, 1881. Souvenirs de la NouvelleCalédonie, Paris, Calman-Lévy.

SARASIn Fritz, 2009. Ethnographie des Kanak de Nouvelle-Calédonie et des Îles Loyauté (19111912), Paris, Ibis Press (traduction de l'édition allemande de 1929).

Saussol Alain, 1979. L'Héritage: essai sur le problème foncier mélanésien en Nouvelle-Calédonie, Paris, Société des Océanistes, Publications de la $\mathrm{SdO} 49$.

Schindlbeck Markus, 2012. Gefunden und Verloren. Arthur Speyer, die dreissiger Jahre und die Verluste der Sammlung Südsee des Ethnologischen Museums Berlin, Berlin, Ethnologisches Museum.

Thode-Arora Hilke, 1989. Für fünfzig Pfennig um die Welt: die Hagenbeckschen Völkerschauen, Francfort, Campus.

-, 1992. Die Familie Umlauff und ihre Firmen - Ethnographica-Händler in Hamburg, Mitteilungen aus dem Museum für Völkerkunde Hamburg 22, pp. 143-158.

—, 1997. Herbeigeholte Ferne. Völkerschauen als Vorläufer exotischer Abenteuerfilme, in Jörg Schöning (éd.), Triviale Tropen. Exotische Reise- und Abenteuerfilme aus Deutschland 1919-1939, Munich, Text + Kritik, pp. 19-33.

-, 2001. Tapa und Tiki: die PolynesienSammlung des Rautenstrauch-Joest-Museums, Cologne, Rautenstrauch-Joest-Museum der Stadt Köln, 2001.

Zelz Caroline Eliot, 1995. Masken aufNeukaledonien, Munster, Lit Verlag.

1. N.B. Pour ne pas allonger inconsidérément cette contribution, on s'est abstenu d'énumérer les sources administratives, militaires, journalistiques utilisées pour ce travail et dont beaucoup sont facilement consultables sur Internet, tel "l'historique " de chacun des navires où navigua Moriceau. 


\title{
ANNEXE : LA CARRIÈre CALÉDONIENNE DE L'ADMINISTRATEUR MORICEAU
}

\author{
(d'après des notes d'Alain Saussol)
}

Il se fait remarquer en juin 1878 en recrutant quelques volontaires (au nombre de huit) pour constituer un petit corps d'éclaireurs. On ne connaît pas les circonstances de cet enrôlement. Le soir du 26 juin, à l'initiative de quelques notables, des Nouméens se réunissent dans un dock et décident de créer un corps de cavaliers qui partiraient le soir même prévenir de l'insurrection les colons disséminés entre Païta et la rivière de la Tontouta. C'est l'agronome Boutan qui va présenter ce projet au gouverneur et obtient son accord. C'est lui qui commande la vingtaine d'hommes qui partent dans la nuit. Il n'est nulle part question de Moriceau (qui a démissionné de la Marine et n'appartient pas encore à l'Administration) ni de Sonneville, dont les initiatives de même nature paraissent être plus tardives.

Moriceau et ses cavaliers sont mentionnés le $1^{\text {er }}$ juillet quand ils passent à Tomo avec les éclaireurs de Boutan. Tous se rendent à Bouloupari. Chemin faisant, ils semblent s'être comportés plutôt durement avec les Kanak de Saint-Vincent parmi lesquels ils ont fait des prisonniers (que les gendarmes leur font relâcher).

Les éclaireurs de Moriceau vont servir à Bouloupari, du $1^{\text {er }}$ au 19 juillet 1878, mais commandés par Saxton, un stockman courageux qui lors d'une opération aura son cheval tué sous lui. Il n'est jamais question de Moriceau qui a dû rentrer vite à Nouméa. Un journaliste britannique note à son sujet :

«Il est venu trois fois ici [à Bouloupari] et a bien pris soin de repartir. Il n'a rien fait. "

Il ne semble pas s'être avéré un foudre de guerre!

En 1881, Moriceau entre dans l'Administration coloniale comme adjoint d'arrondissement. C'est le moment où, après l'insurrection, une administration civile remplace l'Administration militaire. On a besoin d'administrateurs civils pour remplacer les officiers qui, jusque-là, fournissaient les chefs d'arrondissement. Cela a dû faciliter son recrutement. En 1881, il est nommé adjoint du chef $\mathrm{du} 5^{\mathrm{e}}$ arrondissement à Ouégoa. C'est donc là qu'a dû commencer sa carrière administrative.

En 1884, il devient chef d'arrondissement en titre, reste à déterminer où précisément. On sait en revanche qu'en mai 1887, tout juste nommé chef du $4^{\mathrm{e}}$ arrondissement et basé à Touho, il inaugure son poste par un coup d'éclat (que le gouverneur Nouet jugera être une bévue, estimant qu'il avait agi " avec trop de précipitation ") : l'arrestation, pour un motif bénin - un vol de porcs chez un colon -, de Poindi Patchili, ensuite exilé à Obock où il mourra en mai 1888.

En 1889, Moriceau devient administrateur de $1^{\text {ère }}$ classe. C'est aussi l'année de l'Exposition universelle de Paris, où il expose des objets kanak comme il est évoqué. Ensuite, on perd sa trace jusqu'en 1892 où on le retrouve à Houailou comme administrateur $\mathrm{du} 3^{\mathrm{e}}$ arrondissement et, accessoirement, membre " nommé » de la Chambre d'Agriculture (avec des notabilités de l'époque: les Boutan, Jeanneney, Gallet, Camouilly, etc.).

En juillet 1894, Moriceau accompagne le gouverneur Feillet à l'île des Pins, pour proposer aux anciens insurgés de 1878, exilés dans cette île, de revenir sur la Grande Terre moyennant un engagement de travail de cing ans chez les colons. En septembre, deux cents proscrits profitent de l'aubaine.

Le 11 décembre 1894, un nouveau service administratif est créé réunissant Affaires indigènes, Immigration et Office de renseignements dont la direction est confiée à l'administrateur Moriceau. Ce service sera supprimé en janvier 1897 et remplacé par un simple "Bureau des Affaires indigènes et des Renseignements " dépendant directement du gouverneur, et dont Moriceau gardera la direction.

En septembre 1895, le gouverneur Feillet vient à Hienghène inspecter les lieux susceptibles d'accueillir de nouveaux colons. Il y est fraîchement reçu par les Kanak. En octobre 1896, Moriceau vient à nouveau enquêter à Hienghène, alerté par une montée de tension chez les indigènes et d'inquiétude chez les colons. En mars 1897, il revient à Hienghène où, à partir d'une histoire de femmes dit-on, le 6 février, un conflit a éclaté entre gens de Goa et de Bouarate, faisant trois morts. Moriceau doit obtenir le désarmement des Kanak (qui avaient acheté des fusils à des libérés) et une réconciliation. N'y parvenant pas, il demande de pouvoir user de la force et va solliciter un peu partout le concours d'un millier d'auxiliaires comme au bon vieux temps des expéditions militaires. La troupe viendra aussi mais laissera agir les auxiliaires qui mèneront seuls la répression.

Cette action fut dénoncée par l'inspecteur de colonies Arnaud, mais couverte par Le Fol gouverneur par intérim durant l'absence de Feillet car il avait donné son accord à Moriceau et pouvait donc difficilement faire autrement.

Le 4 mai 1897, le ministère des Colonies se préoccupe de l'Exposition universelle de 1900. Une circulaire invite les divers territoires à présenter l'œuvre civilisatrice de la France. Le 28 août 1897 , Moriceau (malade ?) est envoyé en Métropole pour six mois en congé de convalescence. C'est après cette convalescence, peut-être augmentée de ses congés réglementaires, qu'il dut être affecté à Madagascar (où, comme il est signalé ci-dessus, on le retrouve en 1899). C'est donc après le départ de Moriceau de Nouvelle-Calédonie que se tient à Nouméa l'exposition locale de septembre 1899, préparatoire de l'Exposition universelle de 1900 à Paris.

$\mathrm{Au}$ vu de ce qu'on sait aujourd'hui de Moriceau en Nouvelle-Calédonie, il paraît avoir été quelqu'un d'assez remuant, porté à vouloir " en être ", plutôt "mouche du coche" que réellement dynamique, avec un coté un peu " m'as-tu-vu ». 